\title{
Analysis of the Symmetries and Conservation Laws of the Nonlinear Jaulent-Miodek Equation
}

\author{
Mehdi Nadjafikhah and Mostafa Hesamiarshad \\ Department of Mathematics, Islamic Azad University, Karaj Branch, Karaj 31485-313, Iran \\ Correspondence should be addressed to Mostafa Hesamiarshad; mostaf.hesami@gmail.com
}

Received 11 February 2014; Revised 12 April 2014; Accepted 16 April 2014; Published 6 May 2014

Academic Editor: Mariano Torrisi

Copyright (c) 2014 M. Nadjafikhah and M. Hesamiarshad. This is an open access article distributed under the Creative Commons Attribution License, which permits unrestricted use, distribution, and reproduction in any medium, provided the original work is properly cited.

\begin{abstract}
Lie symmetry method is performed for the nonlinear Jaulent-Miodek equation. We will find the symmetry group and optimal systems of Lie subalgebras. The Lie invariants associated with the symmetry generators as well as the corresponding similarity reduced equations are also pointed out. And conservation laws of the J-M equation are presented with two steps: firstly, finding multipliers for computation of conservation laws and, secondly, symbolic computation of conservation laws will be applied.
\end{abstract}

\section{Introduction}

The Jaulent-Miodek equation (J-M) is given by

$$
\begin{gathered}
u_{t}+u_{x x x}+\frac{3}{2} v v_{x x x}+\frac{9}{2} v_{x} v_{x x}-6 u u_{x}-6 u v v_{x}-\frac{3}{2} v^{2} u_{x}=0, \\
v_{t}+v_{x x x}-6 v u_{x}-6 u v_{x}-\frac{15}{2} v^{2} v_{x}=0 .
\end{gathered}
$$

The coupled system of (1) is associated with the J$M$ spectral problem [1]. The relation between this system and Euler-Darboux equation was found by Matsuno [2]. In recent years, much work associated with the J-M equation has been done [3-5]. The symmetry group method plays a fundamental role in the analysis of differential equations. The theory of Lie symmetry groups of differential equations called classical Lie method was first developed by Lie [6] at the end of the nineteenth century. Nowadays, the application of Lie transformations group theory for constructing the solutions of nonlinear partial differential equations (PDEs) is regarded as one of the most active fields of research in the theory of nonlinear PDEs and applications.

Many PDEs in the applied sciences and engineering are continuity equations which express conservation of mass, momentum, energy, or electric charge. Such equations occur in, for example, fluid mechanics, particle and quantum physics, plasma physics, elasticity, gas dynamics, electromagnetism, magnetohydrodynamics, nonlinear optics, and so forth. In the study of PDEs, conservation laws are important for investigating integrability and linearization mappings and for establishing existence and uniqueness of solutions. They are also used in the analysis of stability and global behavior of solutions [7-10].

The present paper is organized as follows. In Section 1, we obtain the symmetry of (1) and Lie symmetry groups of J-M equation are found. In Section 2, we construct the optimal system of one-dimensional subalgebras of (1). Lie invariants and similarity reduced equations corresponding to the infinitesimal symmetries of (1) are obtained in Section 3. In Section 4, the conservation laws of (1) are obtained with finding multipliers, and finally some new conservation laws of (1) are obtained with symbolic computation of conservation laws.

\section{Lie Symmetries of the J-M Equation}

In this section, we draw your attention to the general procedure for determining symmetries for J-M equation; see [11-13]. We consider the one parameter Lie group of 
TABLE 1: The commutator table.

\begin{tabular}{cccc}
\hline$[]$, & $X_{1}$ & $X_{2}$ & $X_{3}$ \\
\hline$X_{1}$ & 0 & 0 & $X_{1}$ \\
$X_{2}$ & 0 & 0 & $\frac{1}{3} X_{2}$ \\
$X_{3}$ & $-X_{1}$ & $-\frac{1}{3} X_{2}$ & 0 \\
\hline
\end{tabular}

infinitesimal transformations on $\left(x^{1}=x, x^{2}=t, u^{1}=u, u^{2}=\right.$ $v)$,

$$
\begin{aligned}
& \tilde{x}=x+s \xi^{1}(x, t, u, v)+O\left(s^{2}\right), \\
& \tilde{t}=x+s \xi^{2}(x, t, u, v)+O\left(s^{2}\right), \\
& \tilde{u}=x+s \varphi^{1}(x, t, u, v)+O\left(s^{2}\right), \\
& \tilde{v}=x+s \varphi^{2}(x, t, u, v)+O\left(s^{2}\right),
\end{aligned}
$$

where $s$ is the group parameter and $\xi^{1}, \xi^{2}, \varphi^{1}$, and $\varphi^{2}$ are the infinitesimals of the transformations for the independent and dependent variables, respectively. The associated vector field is in the following form:

$$
\begin{aligned}
V= & \xi^{1}(t, x, u, v) \partial_{t}+\xi^{2}(t, x, u, v) \partial_{x} \\
& +\varphi^{1}(t, x, u, v) \partial_{u}+\varphi^{2}(t, x, u, v) \partial_{v}
\end{aligned}
$$

The Lie algebra $\mathfrak{g}$ of infinitesimal symmetry of (1) is spanned by three vector fields:

$$
\begin{gathered}
X_{1}=\partial_{t}, \quad X_{2}=\partial_{x}, \\
X_{3}=t \partial_{t}+\frac{1}{3} x \partial_{x}-\frac{2}{3} u \partial_{u}-\frac{1}{3} v \partial_{v} .
\end{gathered}
$$

The commutation relations of the 3-dimensional Lie algebra $\mathfrak{g}$ spanned by the vector fields $X_{1}, X_{2}, X_{3}$ are shown in Table 1 .

Theorem 1. If $u=f(t, x)$ and $v=g(t, x)$ are a solution of $(1)$, then so are the functions

$$
\begin{gathered}
G_{1}(s) \cdot f(t, x)=f(t-s, x), \\
G_{1}(s) \cdot g(t, x)=g(t-s, x), \\
G_{2}(s) \cdot f(t, x)=f(t, x-s), \\
G_{2}(s) \cdot g(t, x)=g(t, x-s), \\
G_{3}(s) \cdot f(t, x)=e^{-(2 / 3) s} f\left(e^{-s} t, e^{-(1 / 3) s} x\right), \\
G_{3}(s) \cdot g(t, x)=e^{-(1 / 3) s} g\left(e^{-s} t, e^{-(1 / 3) s} x\right) .
\end{gathered}
$$

\section{Optimal System of the Jaulent-Miodek Equation}

In this section, we obtain the optimal system and reduced forms of (1) by using symmetry group properties obtained in
TABLE 2: Adjoint representation of the infinitesimal generators.

\begin{tabular}{cccc}
\hline Ad & $X_{1}$ & $X_{2}$ & $X_{3}$ \\
\hline$X_{1}$ & $X_{1}$ & $X_{2}$ & $X_{3}-\varepsilon X_{1}$ \\
$X_{2}$ & $X_{1}$ & $X_{2}$ & $X_{3}-\frac{1}{3} \varepsilon X_{2}$ \\
$X_{3}$ & $e^{\varepsilon} X_{1}$ & $e^{(1 / 3) \varepsilon} X_{2}$ & $X_{3}$ \\
\hline
\end{tabular}

previous section. Since the original partial differential equation has two independent variables, this partial differential equation transforms into the ordinary differential equation after reduction.

A well-known standard procedure [11] allows us to classify all the one-dimensional subalgebras into subsets of conjugate subalgebras. This involves constructing the adjoint representation group, which introduces a conjugate relation in the set of all one-dimensional subalgebras. In fact, for one-dimensional subalgebras, the classification problem is essentially the same as the problem of classifying the orbits of the adjoint representation. Since each one-dimensional subalgebra is determined by nonzero vector in $\mathfrak{g}$, this problem is attacked by the naive approach of taking a general element $V$ in $\mathfrak{g}$ and subjecting it to various adjoint transformations so as to "simplify" it as much as possible. Thus, we will deal with the construction of the optimal system of subalgebras of $\mathfrak{g}$. To compute the adjoint representation, we use the Lie series

$$
\begin{aligned}
\operatorname{Ad}\left(\exp \left(\varepsilon\left(X_{i}\right)\right) X_{j}\right)= & X_{j}-\varepsilon\left[X_{i}, X_{j}\right] \\
& +\frac{\varepsilon^{2}}{2}\left[X_{i},\left[X_{i}, X_{j}\right]\right]-\cdots,
\end{aligned}
$$

where $\left[X_{i}, X_{j}\right]$ is the commutator for the Lie algebra, $\varepsilon$ is a parameter, and $i, j=1,2,3$. Then we have Table 2 .

Theorem 2. An optimal system of one-dimensional Lie algebras of the J-M equation is provided by (1) $X_{3}$, (2) $\alpha X_{1}+X_{2}$, and (3) $X_{1}$, where $\alpha \in \mathbb{R}$ and $\alpha \neq 0$.

Proof. Let $\mathfrak{g}$ be the symmetry group of (1), with adjoint representation determined in Table 2 , and

$$
X=a_{1} X_{1}+a_{2} X_{2}+a_{3} X_{3}
$$

is a nonzero vector field of $\mathfrak{g}$. We will simplify as many of the coefficients of $a_{i}, i=1,2,3$, as possible through judicious applications of adjoint maps to $X$.

Case 1. Suppose first that $a_{3} \neq 0$. Scaling $X$ if necessary, we can assume that $a_{3}=1$. Referring to Table 2, if we act on such a $X$ by $\operatorname{Ad}\left(\exp \left(a_{1} X_{1}\right)\right)$ and $\operatorname{Ad}\left(\exp \left(3 a_{2} X_{2}\right)\right)$, respectively, we can make the coefficients of $v_{1}$ and $X_{2}$ vanish. Thus, every one-dimensional subalgebra generated by a $X$ with $a_{3} \neq 0$ is equivalent to the subalgebra spanned by $X_{3}$.

Case 2. The remaining one-dimensional subalgebras are spanned by vectors of the above form with $a_{3}=0$. If $a_{2} \neq 0$, we can scale to make $a_{2}=1$. Referring to Table 2, we cannot do anything in this case. Thus, every one-dimensional subalgebra generated by a $v$ with $a_{3}=0$ and $a_{2} \neq 0$ is 
equivalent to the subalgebra spanned by $\alpha X_{1}+X_{2}$, where $\alpha$ is arbitrary constant.

Case 3. Consider $a_{3}=0, a_{2}=0$, and $a_{1} \neq 0$. Thus, every onedimensional subalgebra generated by $X$ is equivalent to the subalgebra spanned by $X_{1}$.

\section{Symmetry Reduction of the J-M Equation}

We can now compute the invariants associated with the symmetry operators by integrating the characteristic equations. For example, for the operator characteristic equation $X_{3}=$ $t \partial_{t}+(1 / 3) x \partial_{x}-(2 / 3) u \partial_{u}-(1 / 3) v \partial_{v}$,

$$
\frac{d t}{t}=\frac{3 d x}{x}=-\frac{3 d u}{2 u}=-\frac{3 v}{d v} .
$$

The corresponding invariants are $\lambda=x t^{-1 / 3}, U=u t^{2 / 3}$, and $V=v t^{1 / 3}$. Therefore, solution of our equation in this case is $u=U t^{-2 / 3}, v=V t^{-1 / 3}$. Substituting derivatives of $u$ and $v$ in terms of $\lambda, U$, and $V$ into (1), the coupled system of ordinary differential equation is obtained as follows:

$$
\begin{gathered}
4 U+2 \lambda U_{\lambda}-6 U_{\lambda \lambda \lambda}-9 V V_{\lambda \lambda \lambda}-27 V_{\lambda} V_{\lambda \lambda} \\
+36 U U_{\lambda}+36 U V V_{\lambda}+9 V^{2} U_{\lambda}=0 \\
2 V+2 \lambda V_{\lambda}-6 V_{\lambda \lambda \lambda}+36 V U_{\lambda}+36 U V_{\lambda}+45 V^{2} V_{\lambda}=0 .
\end{gathered}
$$

And for the operator $\alpha X_{1}+X_{2}$, we have

$$
\begin{gathered}
\alpha U_{\lambda}+U_{\lambda \lambda \lambda}+\frac{3}{2} V V_{\lambda \lambda \lambda}+\frac{9}{2} V_{\lambda} V_{\lambda \lambda} \\
-6 U U_{\lambda}-6 U V V_{\lambda}-\frac{3}{2} V^{2} U_{\lambda}=0 \\
\alpha V_{\lambda}+V_{\lambda \lambda \lambda}-6 V U_{\lambda}-6 U V_{\lambda}-\frac{15}{2} V^{2} V_{\lambda}=0,
\end{gathered}
$$

and the corresponding invariants associated with the above operator are $\lambda=x-\alpha t, U=u$, and $V=v$.

\section{Conservation Laws for the J-M Equation}

To deal with the conservation laws, many methods, such as the method based on the Noethers theorem and the multiplier method, are derived by the relationship between the conserved vector of the PDE and the Lie-Bäcklund symmetry generators of the PDE, the direct method, and so forth $[7,8,11]$.

Definition 3. A local conservation law of the PDE system

$$
\Delta_{v}\left(x, u^{(n)}\right)=0, \quad v=1, \ldots, l,
$$

involving $\tilde{x}=x\left(x^{1}, \ldots, x^{p}\right), \tilde{u}=\left(u^{1}, \ldots, u^{q}\right)$, and the derivatives of $u$ with respect to $x$ up to $n$, where $u^{(n)}$ represents all the derivatives of $u$ of all orders from 0 to $n$, is a divergence expression

$$
D_{i} \Phi^{i}[u]=D_{1} \Phi^{1}[u]+\cdots+D_{n} \Phi^{n}[u]=0
$$

holding for all solutions of the system (11). $\Phi^{i}[u]=$ $\Phi^{i}\left(x, u, \partial u, \ldots, \partial_{u}^{r}\right), i=1, \ldots, n$, are called fluxes of the conservation law, and the highest-order derivative $(r)$ present in the fluxes $\Phi^{i}[u]$ is called the order of a conservation law [8].

Remark 4. If one of the independent variables of (11) is time $t$, the conservation law (12) takes the form

$$
D_{t} \Psi[u]+\operatorname{div} \Phi[u]=0,
$$

where $\operatorname{div} \Phi[u]=D_{i} \Phi^{i}[u]=D_{1} \Phi^{1}[u]+\cdots+D_{n} \Phi^{n}[u]$ is a spatial divergence and $x=\left(x^{1}, \ldots, x^{n-1}\right)$ are $n-1$ spatial variables. Here $\Psi[u]$ is referred to as a density, and $\Phi^{i}[u]$ as spatial fluxes of the conservation law (13).

5.1. Computation of Conservation Laws with Finding Multiplier. In this study, we derive the conservation law from the multiplier method. In particular, a set of multipliers $\left\{\Lambda_{\gamma}\right\}_{\nu=1}^{l}=$ $\left\{\Lambda_{v}\left(x, U, \partial U, \ldots, \partial_{U}^{r}\right)\right\}_{\nu=1}^{l}$ yields a divergence expression for the system (11) if the identity

$$
\Lambda_{v}[U] \Delta_{\nu}[U] \equiv D_{i} \Phi^{i}[U]
$$

holds identically for arbitrary functions $U(x)$. Then, on the solutions $U(x)=u(x)$ of the system (11), if $\Delta_{\gamma}[U]$ is nonsingular, one has local conservation law $\Lambda_{\nu}[u] \Delta_{\nu}[u] \equiv$ $D_{i} \Phi^{i}[u]=0$.

Definition 5. The Euler operator with respect to $U^{j}$ is the operator defined by

$$
E_{U^{j}}=\frac{\partial}{\partial U^{j}}-D_{i} \frac{\partial}{\partial U_{i}^{j}}+\cdots+(-1)^{s} D_{i_{1}} \cdots D_{i_{s}} \frac{\partial}{\partial U_{i_{1} \cdots i_{s}}^{j}}+\cdots
$$

for $j=1, \ldots, q[8]$.

Theorem 6. A set of nonsingular local multipliers $\left\{\Lambda_{v}\left(x, U, \partial U, \ldots, \partial_{U}^{r}\right)\right\}_{\nu=0}^{l}$ yields a local conservation law for the system $\Delta_{v}\left(x, u^{(n)}\right)$ if and only if the set of identities

$$
E_{U^{j}}\left(\Lambda_{\nu}\left(x, U, \partial U, \ldots, \partial^{r} U\right) \Delta_{v}\left(x, u^{(n)}\right)\right)=0, \quad j=1, \ldots, q
$$

holds for arbitrary functions $U(x)$ (Theorem 1.3.3, [8]).

The set of (16) yields the set of linear determining equations to find all sets of local conservation law multipliers of the system (11). Now, we consider all local conservation law multipliers of the forms $\Lambda_{1}=\alpha\left(t, x, u, v, u_{t}, v_{t}, u_{x}, v_{x}, u_{t t}, v_{t t}, u_{x x}, v_{x x}\right)$ and 
$\Lambda_{2}=\beta\left(t, x, u, v, u_{t}, v_{t}, u_{x}, v_{x}, u_{t t}, v_{t t}, u_{x x}, v_{x x}\right)$ of (1). The determining equation (16) for J-M equation is

$$
\begin{gathered}
E_{u}\left[\Lambda _ { 1 } \left(u_{t}+u_{x x x}+\frac{3}{2} v v_{x x x}+\frac{9}{2} v_{x} v_{x x}\right.\right. \\
\left.-6 u u_{x}-6 u v v_{x}-\frac{3}{2} v^{2} u_{x}\right) \\
\left.+\Lambda_{2}\left(v_{t}+v_{x x x}-6 v u_{x}-6 u v_{x}-\frac{15}{2} v^{2} v_{x}\right)\right] \equiv 0, \\
E_{v}\left[\Lambda _ { 1 } \left(u_{t}+u_{x x x}+\frac{3}{2} v v_{x x x}+\frac{9}{2} v_{x} v_{x x}\right.\right. \\
\left.\quad-6 u u_{x}-6 u v v_{x}-\frac{3}{2} v^{2} u_{x}\right) \\
\left.+\Lambda_{2}\left(v_{t}+v_{x x x}-6 v u_{x}-6 u v_{x}-\frac{15}{2} v^{2} v_{x}\right)\right] \equiv 0,
\end{gathered}
$$

where $u(x, t)$ and $v(x, t)$ are arbitrary functions. Equation (17) splits with respect to third order derivatives of $u$ to yield the determining PDE system whose solutions are the sets of local multipliers of all nontrivial local conservation laws of the J-M equation.

The solution of the determining system (17) for J-M equation is given by

$$
\begin{aligned}
\alpha=c_{1} x & +6 c_{1} t u+\frac{9}{2} c_{1} t v^{2}+c_{2} u v+c_{3} u_{1 / 6} c_{2} v_{x x} \\
& +\frac{5}{12} c_{2} v^{3}+\frac{3}{4} c_{3} v^{2}+c_{4} v+c_{5}, \\
\beta= & \frac{1}{6} c_{2} u_{x x}+\frac{1}{2} c_{1} x v-\frac{5}{24} c_{2} v_{x}^{2}-\frac{5}{12} v_{x x}-c_{2} V-\frac{3}{2} c_{1} t v_{x x} \\
& -\frac{1}{4} c_{3} v_{x x}+\frac{15}{4} c_{1} t v^{3}+9 c_{1} t u v+\frac{1}{2} c_{2} u^{2}+\frac{5}{4} c_{2} u v^{2} \\
& +\frac{3}{2} c_{3} u v+c_{4} u+\frac{35}{96} c_{2} v^{4}+\frac{5}{8} c_{3} v^{3}+\frac{3}{4} c_{4} v^{2}+\frac{1}{2} c_{5} v+c_{6},
\end{aligned}
$$

where $c_{1}, c_{2}, c_{3}, c_{4}, c_{5}$, and $c_{6}$ are arbitrary constants. So local multipliers are given by

$$
\begin{aligned}
& \text { (1) } \alpha=0, \quad \beta=1, \\
& \text { (2) } \alpha=1, \quad \beta=\frac{1}{2} v, \\
& \text { (3) } \alpha=v, \quad \beta=u+\frac{3}{4} v^{2}, \\
& \text { (4) } \alpha=u+\frac{3}{4} v^{2}, \quad \beta=-\frac{1}{4} v_{x x}+\frac{3}{2} u v+\frac{5}{8} v^{3}, \\
& \text { (5) } \alpha=x+6 t u+\frac{9}{2} t v^{2}, \\
& \beta=\frac{1}{2} x v-\frac{3}{2} t v_{x x}+\frac{15}{4} t v^{3}+9 t u v,
\end{aligned}
$$

$$
\begin{aligned}
& \text { (6) } \alpha=u v-\frac{1}{6} v_{x x}+\frac{5}{12} v^{3} \text {, } \\
& \beta=-\frac{1}{6} u_{x x}-\frac{5}{24} v_{x}^{2}-\frac{5}{12} v v_{x x}+\frac{1}{2} u^{2}+\frac{5}{4} u v^{2}+\frac{35}{96} v^{4} .
\end{aligned}
$$

Multipliers $\alpha$ and $\beta$ determine a nontrivial local conservation law $D_{t} \Psi+D_{x} \Phi=0$ with the characteristic form

$$
\begin{aligned}
D_{t} \Psi+D_{x} \Phi \equiv \alpha & \left(u_{t}+u_{x x x}+\frac{3}{2} v v_{x x x}+\frac{9}{2} v_{x} v_{x x}\right. \\
& \left.\quad-6 u u_{x}-6 u v v_{x}-\frac{3}{2} v^{2} u_{x}\right) \\
+ & \beta\left(v_{t}+v_{x x x}-6 v u_{x}-6 u v_{x}-\frac{15}{2} v^{2} v_{x}\right) .
\end{aligned}
$$

The total divergence operator must be inverted to calculate the conserved quantities $\Phi$ and $\Psi$. To do this, we need to integrate (by parts) one of the expressions in multidimensions involving arbitrary functions and its derivatives, which is a difficult task. The homotopy operator [14] is a powerful and useful algorithmic tool (explicit formula) that originates from homological algebra and variational bicomplexes.

Definition 7. The 2-dimensional homotopy operator is a vector operator with two components, $\left(H_{\mathbf{u}(x, t)}^{(x)} f, H_{\mathbf{u}(x, t)}^{(t)} f\right)$, where

$$
\begin{aligned}
& H_{\mathbf{u}(x, t)}^{(x)} f=\int_{0}^{1}\left(\sum_{j=0}^{q} I_{u^{j}}^{(x)} f\right)[\lambda \mathbf{u}] \frac{d \lambda}{\lambda} \\
& H_{\mathbf{u}(x, t)}^{(t)} f=\int_{0}^{1}\left(\sum_{j=0}^{q} I_{u^{j}}^{(t)} f\right)[\lambda \mathbf{u}] \frac{d \lambda}{\lambda} .
\end{aligned}
$$

The $x$-integrand, $I_{u(x, t)^{j}}^{(x)} f$, is given by

$$
\begin{gathered}
I_{u^{j}}^{(x)} f=\sum_{k_{1}=1}^{M_{1}^{j}} \sum_{k_{2}=0}^{M_{2}^{j}}\left(\sum_{i_{1}=0}^{k_{1}-1} \sum_{i_{2}=0}^{k_{2}} B^{(x)} u_{x^{i_{1}} x^{i_{2}}}^{j}\left(-D_{x}\right)^{k_{1}-i_{1}-1}\right. \\
\left.\times\left(-D_{t}\right)^{k_{2}-i_{2}}\right) \frac{\partial f}{\partial u_{x^{k_{1}} t^{k_{2}}}^{j}}
\end{gathered}
$$

where $M_{1}^{j}, M_{2}^{j}$ are the order of $f$ in $u$ to $x$ and $t$, respectively, with combinatorial coefficient $B^{(x)}=B\left(i_{1}, i_{2}, k_{1}, k_{2}\right)$, where

$$
B\left(i_{1}, i_{2}, k_{1}, k_{2}\right)=\frac{\left(\begin{array}{c}
i_{1}+i_{2} \\
i_{1}
\end{array}\right)\left(\begin{array}{c}
k_{1}+k_{2}-i_{1}-i_{2}-1 \\
k_{1}-i_{1}-1
\end{array}\right)}{\left(\begin{array}{c}
k_{1}+k i_{2} \\
k_{1}
\end{array}\right)} .
$$

Similarly, $t$-integrand, $I_{u(x, t)^{j}}^{(t)} f$, defined as

$$
\begin{array}{r}
I_{u^{j}}^{(t)} f=\sum_{k_{1}=0}^{M_{1}^{j}} \sum_{k_{2}=1}^{M_{2}^{j}}\left(\sum_{i_{1}=0}^{k_{1}} \sum_{i_{2}=0}^{k_{2}-1} B^{(t)} u_{x^{i_{1}} x^{i_{2}}}^{j}\left(-D_{x}\right)^{k_{1}-i_{1}}\right. \\
\left.\quad \times\left(-D_{t}\right)^{k_{2}-i_{2}-1}\right) \frac{\partial f}{\partial u_{x^{k_{1}} t^{k_{2}}}^{j}},
\end{array}
$$

where $B^{(t)}=B\left(i_{2}, i_{1}, k_{2}, k_{1}\right)$. 
We apply homotopy operator to find conserved quantities $\Psi$ and $\Phi$ which yield multipliers $\alpha=0$ and $\beta=1$. We have

$$
\begin{aligned}
f=\alpha\left(u_{t}+u_{x x x}+\frac{3}{2} v v_{x x x}+\frac{9}{2} v_{x} v_{x x}\right. \\
\left.\quad-6 u u_{x}-6 u v v_{x}-\frac{3}{2} v^{2} u_{x}\right) \\
+\beta\left(v_{t}+v_{x x x}-6 v u_{x}-6 u v_{x}-\frac{15}{2} v^{2} v_{x}\right) \\
=v_{t}+v_{x x x}-6 v u_{x}-6 u v_{x}-\frac{15}{2} v^{2} v_{x} .
\end{aligned}
$$

The integrands (22) and (24) are

$$
\begin{aligned}
& I_{u}^{(t)} f=0, \quad I_{u}^{(x)} f=-6 u v, \quad I_{v}^{(t)} f=v, \\
& I_{v}^{(x)} f=-6 u v-\frac{15}{2} v^{3}+v_{x x} .
\end{aligned}
$$

Apply (21) to the integrands (26); therefore

$$
\begin{aligned}
& \Phi=H_{\mathbf{u}(x, t)}^{(x)} f=6 u v-v_{x x}+\frac{5}{2} v^{3}, \\
& \Psi=H_{\mathbf{u}(x, t)}^{(t)} f=-v .
\end{aligned}
$$

So, we have the conservation law of the J-M equation with respect to multipliers $\alpha=0$ and $\beta=1$ :

$$
D_{t}(-v)+D_{x}\left(6 u v-v_{x x}+\frac{5}{2} v^{3}\right)=0
$$

And similarly, conservation laws with respect to other multipliers are given as follows:

(1) $\alpha=1$ and $\beta=(1 / 2) v$ :

$$
\begin{aligned}
& \Phi=-\frac{1}{4} v^{2}-u \\
& \Psi=\frac{15}{16} v^{4}+\frac{9}{2} u v^{2}-2 v v_{x x}-\frac{5}{4} v_{x}^{2}+3 u^{2}-u_{x x}
\end{aligned}
$$

(2) $\alpha=v$ and $\beta=u+(3 / 4) v^{2}$ :

$$
\begin{aligned}
& \Phi=-\frac{1}{4} v^{3}-u v, \\
& \Psi=\frac{9}{8} v^{5}+6 u v^{3}-\frac{9}{4} v^{2} v_{x x}+6 u^{2} v-u v_{x x}-v u_{x x}+u_{x} v_{x}
\end{aligned}
$$

(3) $\alpha=u+(3 / 4) v^{2}$ and $\beta=-(1 / 4) v_{x x}+(3 / 2) u v+(5 / 8) v^{3}$ :

$$
\begin{aligned}
\Phi= & -\frac{5}{32} v^{4}-\frac{3}{4} u v^{2}+\frac{1}{8} v v_{x x}-\frac{1}{2} u^{2}, \\
\Psi= & \frac{25}{32} v^{6}+\frac{39}{8} u v^{4}-\frac{7}{8} v^{3} v_{x x}+\frac{15}{2} v^{2} u^{2}-\frac{3}{2} u v_{x}^{2} \\
& -3 u v v_{x x}-\frac{3}{4} v^{2} u_{x x}+\frac{3}{2} v u_{x} v_{x}+2 u^{3}+\frac{1}{2} u_{x}^{2}+\frac{1}{8} v_{x x}^{2} \\
& -u u_{x x}-\frac{1}{8} v v_{t x}+\frac{1}{8} v_{t} v_{x} ;
\end{aligned}
$$

(4) $\alpha=u v-(1 / 6) v_{x x}+(5 / 12) v^{3}$ and $\beta=-(1 / 6) u_{x x}-$

$(5 / 24) v_{x}^{2}-(5 / 12) v v_{x x}+(1 / 2) u^{2}+(5 / 4) u v^{2}+(35 / 96) v^{4}:$

$$
\begin{aligned}
\Phi= & -\frac{7}{96} v^{5}-\frac{5}{12} u v^{3}+\frac{5}{72} v v_{x}^{2}-\frac{1}{2} v u^{2} \\
& +\frac{5}{36} v^{2} v_{x x}=\frac{1}{12} v u_{x x}+\frac{1}{12} u v_{x x} \\
\Psi= & \frac{25}{64} v^{7}+\frac{45}{16} u v^{5}-\frac{25}{48} v^{3} v_{x}^{2}+\frac{23}{4} v^{3} u^{2}-\frac{95}{96} v^{4} v_{x x} \\
& -\frac{11}{4} u v^{2} v_{x x}-\frac{5}{12} v^{3} u_{x x}+3 v u^{3}-\frac{5}{4} u v v_{x}^{2}-\frac{1}{2} u^{2} v_{x x} \\
& -\frac{5}{36} v^{2} v_{t x}+\frac{5}{36} v v_{t} v_{x}+\frac{1}{3} v v_{x x}^{2}+\frac{5}{24} v_{x}^{2} v_{x x}-u v u_{x x} \\
& +\frac{1}{12} u_{t} v_{x}-\frac{1}{12} v u_{t x}+\frac{1}{12} v_{t} u_{x}+\frac{1}{6} u_{x x} v_{x x}-\frac{1}{12} u v_{t x}
\end{aligned}
$$

(5) $\alpha=x+6 t u+(9 / 2) t v^{2}$ and $\beta=(1 / 2) x v-(3 / 2) t v_{x x}+$ $(15 / 4) t v^{3}+9 t u v$ :

$$
\begin{aligned}
\Phi= & -\frac{15}{16} t v^{4}-\frac{9}{2} t u v^{2}+\frac{3}{4} t v v_{x x}-\frac{1}{4} x v^{2}-3 t u^{2}-x u \\
\Psi= & \frac{75}{16} t v^{6}+\frac{117}{4} t u v^{4}+\frac{15}{16} x v^{4}+45 t u^{2} v^{2}-\frac{21}{2} t v^{3} v_{x x} \\
& +9 t v u_{x} v_{x}-9 t u v_{x}^{2}+\frac{9}{2} x u v^{2}-18 t u v v_{x x}-\frac{9}{2} t v^{2} u_{x x} \\
& +12 t u^{3}+\frac{5}{4} v v_{x}+\frac{3}{4} t v_{x x}^{2}-2 x v v_{x x}+\frac{3}{4} t v_{t} v_{x}-6 t u u_{x x} \\
& +3 x u^{2}-\frac{5}{4} x v_{x}^{2}+3 t u_{x}^{2}-\frac{3}{4} t v v_{t x}-x u_{x x}+u_{x} .
\end{aligned}
$$

5.2. Symbolic Computation for Finding Conservation Laws Equation. This subsection covers the application of the homotopy operator to the computation of conservation laws of J-M equation. Finding a conservation law needs computing the density $\Psi$ first, followed by computing of the flux $\Phi$. Computing flux $\Phi$ will require using homotopy operator. Following the approach by Hereman et al. $[9,14,15]$, a candidate density is built as a linear combination (with undetermined coefficients of differential terms) which is invariant under the scaling symmetry of the given PDE. By determining $\Psi$ we can compute $D_{t} \Psi$ and remove all time derivatives; $D_{t} \Psi$ must be a divergence. Thus, using Theorem 4.4 of [10], one requires that

$$
E_{U^{j}}\left(D_{t} \Psi\right)=0, \quad j=1, \ldots, N .
$$

This leads to a linear system for the undetermined coefficients. Substituting its solution into the candidate for $\Psi$ gives the actual density. Finally, the $\Phi=\operatorname{div}^{-1}\left(D_{t} \Psi\right)$ is computed with the homotopy operator.

Jaulent-Miodek equation is invariant under the scaling (dilation) symmetry (4):

$$
(t, x, u, v) \longrightarrow\left(\lambda^{3} t, \lambda x, \lambda^{-2} u, \lambda^{-1} v\right) .
$$


Conservation law (13) must hold on solutions of (1). Therefore, we search for polynomial conservation laws that obey the scaling symmetry of the PDE. Indeed, we have to find a polynomial conservation law that does not adhere to the scaling symmetry. We choose a scaling factor for one of the components of (13). The selected scaling factor will be called the rank $(R)$ of that component. Then, we construct a candidate for that component as a linear combination of monomial terms (all of rank $R$ ) with undetermined coefficients. If we remove divergence and divergence-equivalent terms dynamically that candidate will be shorted and of low order.

For J-M equation we will compute the density $\Psi$ of a fixed rank; for example, $R=-3$. We construct a list of differential terms which contains all powers of dependent variables and their derivatives and products of them of rank -3 :

$$
\begin{aligned}
Q=\{ & u_{x}^{3}, v^{3}, t u^{3}, x u^{2}, u u_{x}, u v, u^{2} u_{x x x}, u_{x}^{3} u_{x x}, v^{3} u_{x x}, v u_{x}^{2}, \\
& v_{x} u_{x}^{3}, v^{3} v_{x}, t u^{3} u_{x x}, x u^{2} u_{x x}, u u_{x} u_{x x}, u v u_{x x} \\
& u^{2} u_{x x} u_{x x x}, v u^{2} u_{x x}, v^{2} u_{x} u_{x x}, t u^{3} v_{x}, x u^{2} v_{x}, u v_{x} u_{x} \\
& \left.u v v_{x}, u^{2} v_{x} u_{x x x}, v v_{x} u_{x}^{2}, v^{2} u_{x} v_{x}\right\}
\end{aligned}
$$

By removing all terms that are divergences or divergenceequivalent to other terms in $Q$, we have

$$
\begin{aligned}
Q=\{ & u_{x}^{3}, v^{3}, t u^{3}, x u^{2}, v^{2} u_{x}, v^{3} u_{x x}, v u_{x}^{2}, v_{x} u_{x}^{3}, \\
& t u^{3} u_{x x}, x u^{2} u_{x x}, u v u_{x x}, u^{2} u_{x x} u_{x x x}, v u_{x}^{2} u_{x x}, \\
& v^{2} u_{x} u_{x x}, t u^{3} v_{x}, x u^{2} v_{x}, u u_{x} v_{x}, u^{2} v_{x} u_{x x x}, \\
& \left.v v_{x} u_{x}^{2}, v^{2} v_{x} u_{x}\right\} .
\end{aligned}
$$

Now, by forming a candidate density combining the terms in $Q$ linearly with undetermined coefficients $c_{i}$,

$$
\begin{aligned}
\Psi=c_{1} & u_{x}^{3}+c_{2} v^{3}+c_{3} t u^{3}+c_{4} x u^{2}+c_{5} v^{2} u_{x}+c_{6} v^{3} u_{x x} \\
& +c_{7} v u_{x}^{2}+c_{8} v_{x} u_{x}^{3}+c_{9} t u^{3} u_{x x}+c_{10} x u^{2} u_{x x} \\
& +c_{11} u v u_{x x}+c_{12} u^{2} u_{x x} u_{x x x}+c_{13} v u_{x}^{2} u_{x x} \\
& +c_{14} v^{2} u_{x} u_{x x}+c_{15} t u^{3} v_{x}+c_{16} x u^{2} v_{x}+c_{17} u u_{x} v_{x} \\
& +c_{18} u^{2} v_{x} u_{x x x}+c_{19} v v_{x} u_{x}^{2}+c_{20} v^{2} v_{x} u_{x} .
\end{aligned}
$$

Compute the total derivative with respect to $t$ of (38), and set

$$
F=-D_{t} \Psi
$$

After replacing $u_{t}$ with $-u_{x x x}-(3 / 2) v v_{x x x}-(9 / 2) v_{x} v_{x x}+$ $6 u u_{x}+6 u v v_{x}+(3 / 2) v^{2} u_{x}$ and $v_{t}$ by $-v_{x x x}+6 v u_{x}+6 u v_{x}+$ $(15 / 2) v^{2} v_{x}$, (39) must be a divergence, use (34), and require

$$
E_{u(t, x)} F=0, \quad E_{v(x, t)} F=0 .
$$

The solution of system (40) is

$$
\begin{aligned}
& c_{1}=0, \quad c_{2}=0, \quad c_{3}=0, \quad c_{4}=0, \\
& c_{5}=0, \quad c_{6}=c_{20}, \quad c_{7}=c_{11}, \quad c_{8}=\frac{1}{3} c_{13}, \\
& c_{9}=0, \quad c_{10}=0, \quad c_{12}=0, \quad c_{14}=c_{19}, \\
& c_{15}=0, \quad c_{16}=0, \quad c_{17}=c_{11}, \quad c_{18}=0,
\end{aligned}
$$

where $c_{11}, c_{13}, c_{19}$, and $c_{20}$ is arbitrary.

Case 1. Substitute (41) and $c_{11}=1, c_{13}=0, c_{19}=0$, and $c_{20}=0$ into (38) and (39) given $\Psi=v u_{x}^{2}+u v u_{x x}+u u_{x} v_{x}$ and

$$
\begin{aligned}
F=u_{x} v_{x} u_{x x x}+2 v u_{x} u_{x x x x}+\frac{9}{2} u_{x} v_{x}^{2} v_{x x}+9 v u_{x} v_{x x}^{2} \\
+u u_{x} v_{x x x x}+3 v^{2} u_{x} v_{x x x x}+\frac{9}{2} u v_{x} v_{x x}+u v_{x} u_{x x x x} \\
+6 u v_{x}^{2} v_{x x x}+\frac{3}{2} u v^{2} v_{x x x x x}+\frac{3}{2} v^{2} u_{x x} v_{x x x}+v u_{x x} u_{x x x} \\
+v u u_{x x x x x}+u u_{x x} v_{x x x}-12 u^{2} v_{x} u_{x x}-\frac{9}{2} v^{3} u_{x} u_{x x} \\
-\frac{3}{2} u v^{3} u_{x x x}-6 u^{2} v^{2} v_{x x x}-6 v u^{2} u_{x x x}-6 u^{2} u_{x} v_{x x} \\
-30 u u_{x}^{2} v_{x}-27 v^{2} v_{x} u_{x}^{2}+u_{x}^{2} v_{x x x}-6 u^{2} v_{x}^{3}-18 v u_{x}^{3} \\
+\frac{27}{3} v u_{x} v_{x} v_{x x x}+\frac{9}{2} v v_{x} v_{x x} u_{x x}+9 u v v_{x} v_{x x x x} \\
+15 u v v_{x x} v_{x x x}-\frac{69}{2} u v^{2} u_{x} v_{x x}-27 u v^{2} v_{x} u_{x x} \\
-48 u v u_{x} u_{x x}-57 u v u_{x} v_{x}^{2}-24 v u^{2} v_{x} v_{x x} .
\end{aligned}
$$

Since $F=\operatorname{div} \Phi$, the flux $\Phi$ can be computed with the 1D homotopy operator which inverts divergences. Applying 1D homotopy operator formulas in (21) and removing curl term of flux $\Phi$ yield

$$
\begin{aligned}
\Phi=- & \frac{3}{2} v^{3} u_{x}^{2}-\frac{3}{2} u v^{3} u_{x x}-6 v^{2} u^{2} v_{x x}-\frac{45}{2} u v^{2} u_{x} v_{x} \\
& -18 u v u_{x}^{2}-\frac{3}{2} v^{2} u_{x} v_{x x x}+\frac{9}{2} v v_{x} u_{x} u_{x x}+\frac{3}{2} u v^{2} v_{x x x x} \\
& -6 v u^{2} u_{x x}+6 u v v_{x} v_{x x x}+\frac{9}{2} u v v_{x x}^{2}-6 u^{2} u_{x} v_{x} \\
& +v u_{x} u_{x x x}+u v u_{x x x x}+u u_{x} v_{x x x} .
\end{aligned}
$$

Case 2. Substitutec $c_{11}=0, c_{13}=1, c_{19}=0$, and $c_{20}=0$ into (38), given

$$
\begin{aligned}
& \Psi=\frac{1}{3} v_{x} u_{x}^{3}+v u_{x}^{2} u_{x x}, \\
& \Phi=-\frac{1}{6} u_{x}^{2}\left(36 u v^{2} v_{x x}+9 v^{3} u_{x x}+69 v^{2} u_{x} v_{x}\right.
\end{aligned}
$$




$$
\begin{aligned}
& +36 u v v_{x}^{2}-36 v v_{x} v_{x x x}+12 u u_{x} v_{x} \\
& -27 v v_{x x}^{2}+36 u v u_{x x}+48 v u_{x}^{2} \\
& \left.-9 v^{2} v_{x x x x}-6 v u_{x x x x}-2 u_{x} v_{x x x}\right)
\end{aligned}
$$

Case 3. Substitute $c_{11}=0, c_{13}=0, c_{19}=1, c_{20}=0$,

$$
\begin{aligned}
\Psi=v^{2} u_{x} u_{x x}+ & v v_{x} u_{x}^{2}, \\
\Phi=-\frac{1}{2} v u_{x}( & 12 u v^{2} v_{x x}+3 v^{3} u_{x x}+33 v^{2} u_{x} v_{x} \\
& +12 u v v_{x}^{2}-12 v v_{x} v_{x x x}+12 u v u_{x x} \\
& +12 u u_{x} v_{x}-3 v^{2} v_{x x x x}+24 v u_{x}^{2}-9 v v_{x x}^{2} \\
& \left.-2 v u_{x x x x}-2 u_{x} v_{x x x}\right) .
\end{aligned}
$$

And finally, $c_{11}=0, c_{13}=0, c_{19}=0, c_{20}=1$,

$$
\begin{aligned}
& \Psi=\frac{1}{3} v^{3} u_{x x}+ v^{2} u_{x} v_{x} \\
& \Phi=-\frac{1}{6} v^{2}\left(3 v^{3} u_{x x}+12 u v^{2} v_{x x}+63 v^{2} v_{x} u_{x}\right. \\
&+12 u v v_{x}^{2}+48 v u_{x}^{2}-9 v v_{x x}^{2} \\
&-12 v v_{x} v_{x x x}+36 u u_{x} v_{x}-3 v^{2} v_{x x x x} \\
&\left.+12 u v u_{x x}-2 v u_{x x x x}-6 u_{x} v_{x x x}\right) .
\end{aligned}
$$

\section{Conclusion}

In this paper, we studied Jaulent-Miodek equation using the Lie symmetry group of infinitesimal transformations of the equation. We found that the underlying equation admits a three-dimensional Lie algebra. We obtained the optimal system of one-dimensional subalgebras of the Lie algebra of the equation. These subalgebras were then used to reduce the underlying equation to nonlinear third order ordinary system of differential equations. Further conservation laws are constructed for this equation in two methods. First, conservation laws of the equation are obtained by finding multipliers; then some other conservation laws of J-M equation are obtained with symbolic computation of conservation laws.

\section{Conflict of Interests}

The authors declare that there is no conflict of interests regarding the publishing of this paper.

\section{References}

[1] M. Jaulent and I. Miodek, "Nonlinear evolution equations associated with 'energy-dependent Schrödinger potentials,' Letters in Mathematical Physics, vol. 1, no. 3, pp. 243-250, 1976.

[2] Y. Matsuno, "Reduction of dispersionless coupled Kortewegde Vries equations to the Euler-Darboux equation," Journal of Mathematical Physics, vol. 42, no. 4, pp. 1744-1760, 2001.
[3] E. Fan, "Uniformly constructing a series of explicit exact solutions to nonlinear equations in mathematical physics," Chaos, Solitons and Fractals, vol. 16, no. 5, pp. 819-839, 2003.

[4] A. Mohebbi, Z. Asgari, and M. Dehghan, "Numerical solution of nonlinear Jaulent-Miodek and Whitham-Broer-Kaup equations," Communications in Nonlinear Science and Numerical Simulation, vol. 17, no. 12, pp. 4602-4610, 2012.

[5] J. Biazar and M. Eslami, "Homotopy analysis method for nonlinear Jaulent-Miodek equation," Journal of Information and Computing Science, vol. 5, no. 2, pp. 083-088, 2010.

[6] S. Lie, "On integration of a class of linear partial differential equations by means of definite integrals," Archiv der Mathematik, vol. 6, p. 328, 1881, Translation by N. H. Ibragimov.

[7] G. Bluman, "New conservation laws obtained directly from symmetry action on a known conservation law," Journal of Mathematical Analysis and Applications, vol. 322, no. 1, pp. 233250, 2006.

[8] G. W. Bluman, A. F. Cheviakov, and S. C. Anco, Applications of Symmetry Methods to Partial Differential Equations, vol. 168 of Applied Mathematical Sciences, Springer, New York, NY, USA, 2010.

[9] W. Hereman, "Symbolic computation of conservation laws of nonlinear partial differential equations in multi-dimensions," International Journal of Quantum Chemistry, vol. 106, pp. 278299, 2006.

[10] D. Poole and W. Hereman, "The homotopy operator method for symbolic integration by parts and inversion of divergences with applications," Applicable Analysis, vol. 89, no. 4, pp. 433455, 2010.

[11] P. J. Olver, Applications of Lie Groups to Differential Equations, vol. 107 of Graduate Texts in Mathematics, Springer, New York, NY, USA, 2nd edition, 1993.

[12] G. W. Bluman and J. D. Cole, Similarity Methods for Differential Equations, vol. 13 of Applied Mathematical Sciences, Springer, New York, NY, USA, 1974.

[13] G. W. Bluman and S. Kumei, Symmetries and Differential Equations, vol. 81 of Applied Mathematical Sciences, Springer, New York, NY, USA, 1989.

[14] W. Hereman, M. Colagrosso, R. Sayers et al., "Continuous and discrete homotopy operators and the computation of conservation laws," in Differential Equations with Symbolic Computation, D. Wang and Z. Zheng, Eds., pp. 249-285, Birkhäuser, Basel, Switzerland, 2005.

[15] L. D. Poole, Symbolic computation of conservation laws of nonlinear partial differential equations using homotopy operators [Ph.D. thesis], Colorado School of Mines, 2009. 


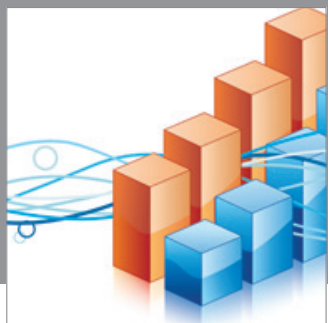

Advances in

Operations Research

mansans

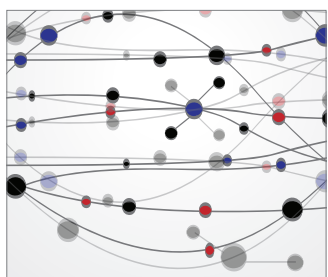

The Scientific World Journal
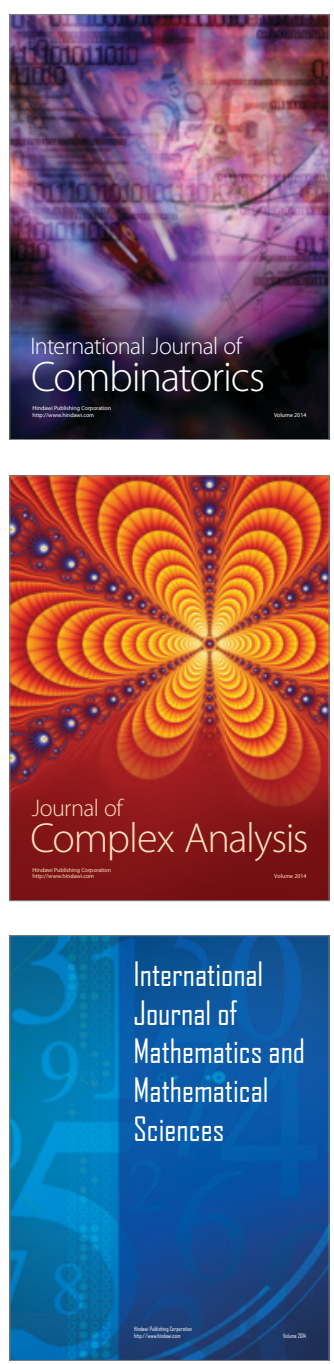
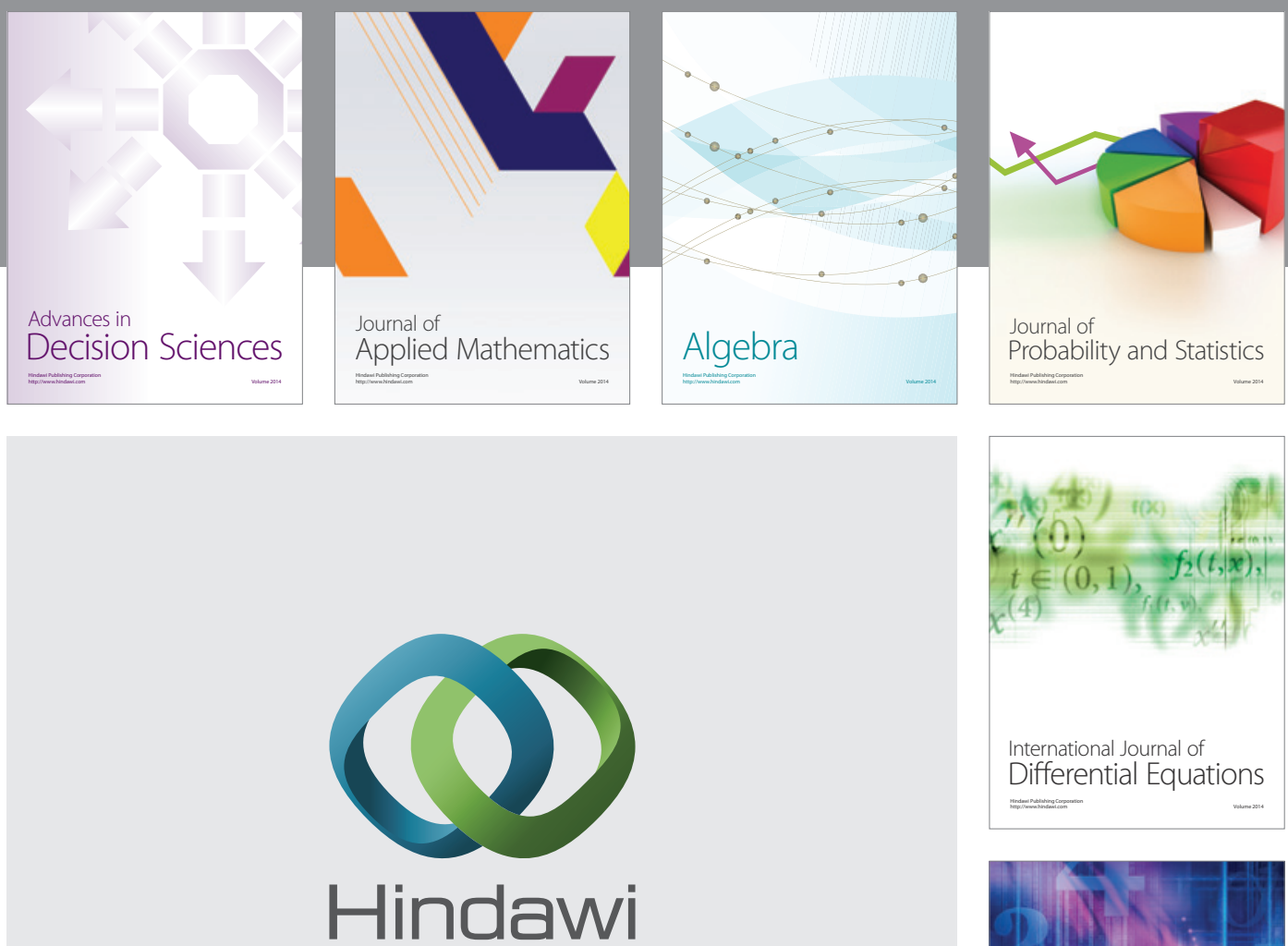

Submit your manuscripts at http://www.hindawi.com
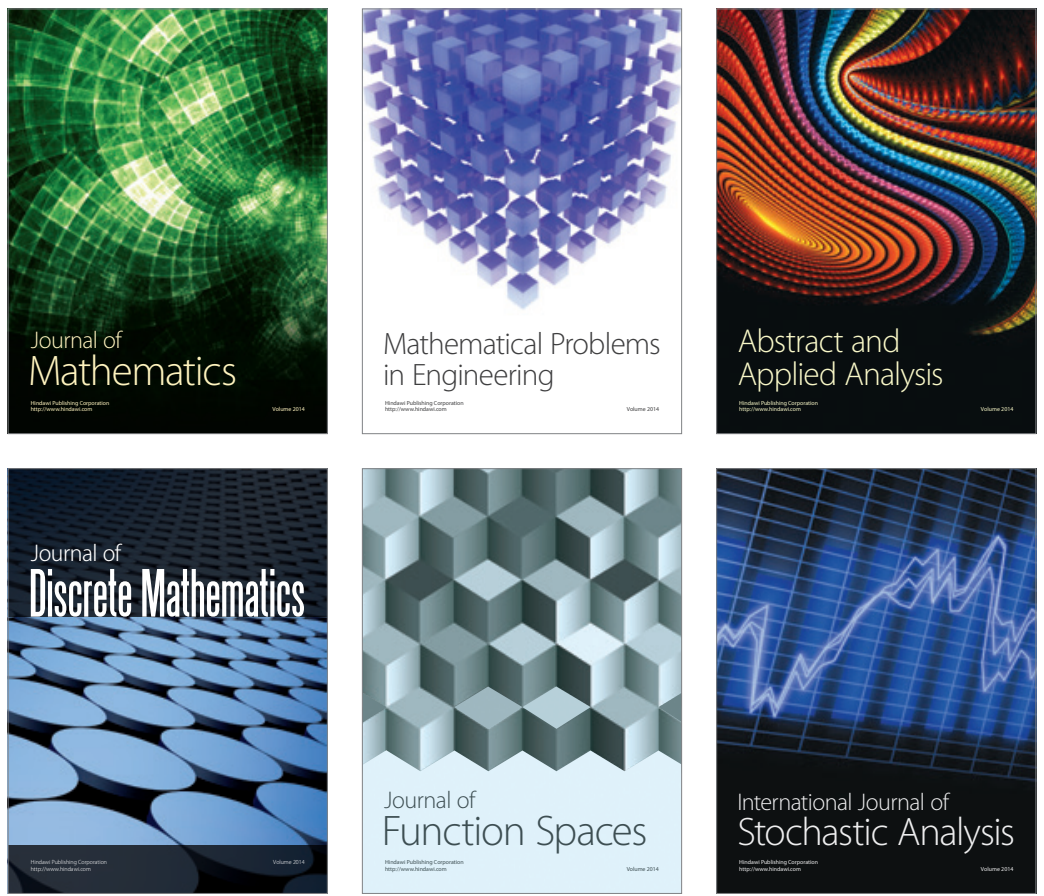

Journal of

Function Spaces

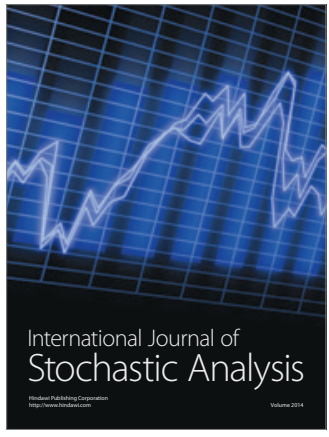

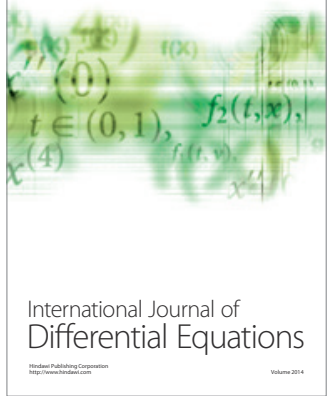
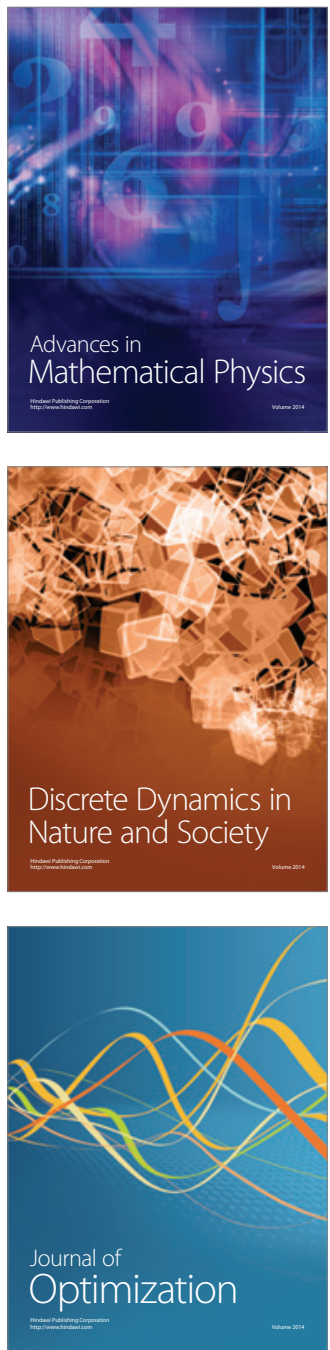